\title{
Optical Recording Analysis of Olfactory Response of the Procerebral Lobe in the Slug Brain
}

Tetsuya Kimura, ${ }^{1,4}$ Shoichi Toda, ${ }^{2}$ Tatsuhiko Sekiguchi, ${ }^{1}$ Shigenori Kawahara, ${ }^{3}$ and Yutaka Kirino ${ }^{3}$

${ }^{1}$ Tsukuba Research Center,

SANYO Electric Co. Ltd.,

Tsukaba, Ibaraki 305, Japan

${ }^{2}$ Faculty of Pharmaceutical Sciences,

Kyushu University,

Fukuoka, Fukuoka 812, Japan

${ }^{3}$ School of Pharmaceutical Sciences,

The University of Tokyo,

Hongo, Tokyo 113, Japan

\begin{abstract}
We studied the oscillatory properties and the olfactory responses of the procerebral (PC) lobe of the cerebral ganglion in the terrestrial mollusc Limax marginatus. The PC lobe, a central olfactory organ in Limax, is a highly interconnected network of local interneurons that receives olfactory inputs from the inferior and superior tentacular noses. We used an optical recording technique with a voltage-sensitive dye to record the activity of the PC lobe from either the posterior or the dorsal surface. The recordings revealed that almost all PC interneurons showed spontaneous oscillatory activities that had been entrained with each other. Upon presentation to the nose of odors to which the slugs had been aversively conditioned, the basal level of the oscillation changed biphasically. In the early phase of the response, depolarization in the basal level of the oscillation occurred in one or more belt-shaped regions parallel to the dorsoventral axis. In the late phase of the response, hyperpolarization of basal potential level of the PC lobe oscillations occurred in a wider area. Such spatial and temporal modulation was not observed when the unpaired control odors were

${ }^{4}$ Corresponding author.
\end{abstract}

presented to the preparation, whereas the same preparations responded to the aversively conditioned stimuli. Thus, it was considered that the spatial and temporal response in the basal level of oscillation was specific to the aversively conditioned odors. Furthermore, the spatial pattern of the depolarization modulation in the early phase was repeatable in multiple trials performed using the same odor, although different odors produced different spatial patterns of the modulation. From these results, we conclude that in the PC lobe learned odors are represented as spatial and temporal activity patterns of oscillators that constitute a coherent network.

\section{Introduction}

Oscillatory phenomena have been detected in the CNSs of many species of animals and are considered to contribute to information processing and motor control. For example, Freeman and Schneider (1982) reported that odor stimuli induce spatially and temporally patterned neural activity in the rabbit olfactory bulb and that the neural activity patterns change upon conditioning. It was also reported that activities of cortical neurons in the primary visual area of the cat, which have different receptive fields, are entrained with each other when they respond to different parts of an object (Gray et al. 1989). Moreover, oscillatory phenomena similar to those in mammalian brains

LEARNING \& MEMORY 4:389-400 @ 1998 by Cold Spring Harbor Laboratory Press ISSN1072-0502/98 \$5.00

$$
\begin{array}{lllllllllllllll}
\hline & E & A & R & N & I & N & G & \underset{389}{\boldsymbol{Q}} & M & E & M & O & R & Y
\end{array}
$$


have been detected in the olfactory systems of invertebrates. Oscillation recorded from glomeruli in the locust antennal lobe became entrained with each other when odors were applied to the antenna (Laurent and Davidowitz 1994; Laurent and Naragi 1994). In mollusks, the interneurons of the procerebral (PC) lobe, into which olfactory afferent fibers project, exhibit spontaneous membrane potential oscillation (Gelperin and Tank 1990; Delaney et al. 1994). These experimental findings suggest that oscillatory circuits have general functions in olfactory processing in the brain.

The oscillatory circuit of the PC lobe is considered to be involved in both olfactory recognition and odor learning. The frequency of PC lobe oscillation is modulated by application of odors to the superior (Delaney et al. 1994; Gervais et al. 1996) and the inferior tentacular nose (Kimura et al. 1998a). Interestingly, application of aversive-conditioned odors but not of control odors to the inferior tentacular nose induces a decrease in oscillatory frequency in the PC lobe (Kimura et al. 1998a). Therefore, it is considered that the response of PC interneurons to odors applied to the inferior tentacular nose is influenced by prior olfactory experience. Moreover, by use of a fluorescent dye as an activity marker, our study revealed that the activity of some PC interneurons increases upon odor-taste associative conditioning and showed that they usually form a belt-shaped cluster along the dorsoventral (D-V) axis (Kimura et al. 1998b). One belt-shaped cluster was detected on the PC lobe when the slug learned about one odor, and two clusters were detected on the PC lobe when the slug learned about two odors. From these findings, it is considered that the PC interneurons forming a belt-shaped cluster are related to formation of each odor memory.

Recently, optical recording studies revealed many interesting properties of PC lobe activity in slugs (e.g., see Gelperin et al. 1993; Kleinfeld et al. 1994; Gervais et al. 1996; Kawahara et al. 1997). In the present study, using an optical recording technique, we analyzed the olfactory responses of PC interneurons to aversively conditioned odors that induced a decrease in the PC oscillatory frequency.

\section{Materials and Methods}

\section{CONDITIONING AND SCREENING \\ OF EXPERIMENTAL ANIMALS}

The laboratory-cultured slugs (Limax margin- atus) were placed individually in small plastic containers and kept there for 1 day without food. The slugs are usually strongly attracted to carrot and cucumber odors. When carrot or cucumber juice as an odor source was placed in front of the slugs' heads they approached it and began exhibiting a biting movement of the lip after touching the odor source (eating behavior) (Kimura et al. 1998a). On the other hand, direct application of a solution of quinidine sulfate (Yamada et al. 1992) to the lip induced a strong aversive effect, as evidenced by an acute turn in the crawling direction of the slug. In aversive training (food odor), the slugs were exposed to carrot or cucumber odor as a conditioned stimulus (CS), followed immediately by application of the quinidine sulfate solution as an unconditioned stimulus (UCS). It was reported previously that this training causes slugs to avoid the CS odor source and exhibit eating behavior at a lower frequency (Kimura et al. 1998a). Such behavioral changes induced by CS-UCS association do not occur when the CS-UCS interval is 5 min or longer (unpaired training) (Kimura et al. 1998a). In the present study, we performed two types of conditioning procedures (two-odor conditioning and differential conditioning) using two odors (carrot and cucumber) as $\mathrm{CS}_{1}$ and $\mathrm{CS}_{2}$ and quinidine sulfate solution as a UCS. The methods of conditioning are described in detail elsewhere (Kimura et al. $1998 a)$. In the first procedure slugs received aversive training twice with each of the two odors. In the differential conditioning, the slugs were subjected to aversive training with one odor twice and unpaired training with the other odor twice. One day after conditioning, the level of preference (in terms of frequency of the occurrence of eating behavior) of each animal for $\mathrm{CS}_{1}$ and $\mathrm{CS}_{2}$ was examined to select slugs to be used in the physiological study. The methods for behavioral testing and selection of experimental animals are described in detail elsewhere (Kimura et al. 1998a).

\section{OPTICAL MEASUREMENT OF THE PC ACTIVITY}

One or two days after conditioning, each slug was injected with an anesthetizing solution (Kimura et al. 1998a) into the body cavity, after which the cerebral ganglion with the inferior tentacle (random choice of right and left nose) was dissected from the body in an anesthetic solutionfilled dish. The dissected preparation was then placed into an anesthetic solution-filled experimental chamber, which consisted of two compart- 
ments with a slit connecting them (see Fig. 2). One of the compartments (ganglion compartment) had a glass bottom that allowed for observation of the fluorescence, and the other (nose compartment) had an inclined bottom for easy exposure of the olfactory epithelium of the inferior tentacular nose to the air.

After placement of the preparation in the ganglion compartment, the inferior nose was moved gently into the nose compartment. After positioning of the nose and ganglion, the anesthetizing solution was removed from both compartments and a slit connecting the compartments was sealed with Vaseline to prevent the mixing of solutions in the two compartments. Following this, both compartments were filled with normal slug saline, and then only the cerebral ganglion was rinsed with a fluorescent voltage-sensitive dye, DI-4-ANEPPS (Molecular Probes), diluted with normal slug saline $(0.02 \%)$ at $20^{\circ} \mathrm{C}$.

Twenty-five minutes later, the voltage-sensitive dye was flushed out several times with normal saline solution. The ganglion and nose were positioned appropriately for viewing of fluorescence of the PC lobe, and then the ganglion was rinsed with $4 \%$ low-melting-point agarose (Sigma) that had been dissolved in normal saline to fix it in position.

The chamber was then left standing for $16 \mathrm{~min}$ to allow polymerization of the agarose and subsequently mounted on the stage of a fluorescence microscope (Olympus IX70) equipped with a 10x objective lens (NA 0.40), a band-pass excitation filter (530-550 nm), a dichroic mirror (565 nm), and a long-pass emission filter $(580 \mathrm{~nm})$. The chamber was connected to tubes for perfusion with normal saline. In our setup, the two compartments were differentially perfused $(2 \mathrm{ml} / \mathrm{min})$, and the surface level of the saline in the nose compartment was changed to expose the olfactory sensory epithelium to the air.

PC lobe activity was determined by using electrophysiological measurement of the local field potential (LFP) and optical recording techniques. For monitoring of spontaneous PC lobe activity, the LFP was measured from the surface of the internal mass (a mass of neuropil located in an anteromedial part of the PC lobe) using a glass pipette (tip diam. $=10 \mu \mathrm{m}$ ) filled with normal saline. The recorded LFP signals were amplified by an amplifier with a band-pass filter $(0.08-30 \mathrm{~Hz})$, and the amplified signals were stored using a thermal array recorder. Although the oscillatory frequency of PC lobe activity fluctuated immediately after setup, the extent of the fluctuation decreased gradually until the oscillatory frequency became constant. We usually waited for at least $30 \mathrm{~min}$ after setup before starting the odor application experiments.

Olfactory responses of the PC lobe were examined using the optical recording technique described above. The optical measurement was completed within 40-60 sec during each experimental session, in which response to an odor was examined. When an odor was applied, $1.5 \mathrm{~min}$ before the start of optical measurements the olfactory epithelium was exposed to the air by lowering the surface level of the saline in the nose compartment. Exposure to odors was performed through use of a glass pipette, the tip of which was positioned above the nose. This olfactory stimulation system is similar to that described elsewhere (Kimura et al. 1998a). The nose was exposed to a constant airflow $(30 \mathrm{ml} / \mathrm{min})$, and we could apply odors without changing the flow rate.

Fluorescence from the hemiganglion was recorded using a photodiode array (HR Deltaron 1700, Fuji Film Co. Ltd.) that was controlled by a DOS/V computer. This photodiode array can be used to acquire consecutive images of an entire field with a typical resolution at a magnification of $6 \mu \mathrm{m} /$ pixel for a $128 \times 128$ image at a frame rate of $26 \mathrm{~Hz}$, which we used. Temporal changes in fluorescence intensity were considered to contain components related to slight photo bleaching and ones related to alterations in membrane potential. The photo-bleaching component was compensated for using the function

$$
\begin{aligned}
F_{\mathrm{e}}(x, y, t)= & k_{\mathrm{o}}(x, y) \times \exp \left[\mathrm{k}_{1}(x, y) \times t\right]+k_{2}(x, y) \\
& \times \exp \left[k_{3}(x, y) \times t\right]
\end{aligned}
$$

where $F_{\mathrm{e}}(t, x, y)$ is the estimated fluorescence intensity at time $t$ at location $(x, y)$, and $k_{0}-k_{3}(x, y)$ are constants at location $(x, y)$. The exponential terms are related to photo bleaching of the dye that dissolved in the cell membrane and that which did not, respectively. We used the Levenberg-Maruadt algorithm to estimate $k_{0}-k_{3}$ as those values that minimized $\chi^{2}$. The data used for the fitting were collected in 4-10 oscillatory cycles immediately before the onset of stimulation and after the end of stimulation. The corrected fluorescence intensity $\left(F_{\mathrm{c}}\right)$ was calculated as

$$
F_{\mathrm{c}}(x, y, t)=f(x, y, t)-\mathrm{F}_{\mathrm{e}}(x, y, t)
$$

where $f(x, y, t)$ is the fluorescence intensity of a pixel located at $(x, y)$ at a given time $t$.

Fractional change of the fluorescence intensity 
$(\Delta F / F)$ related to a change in membrane potential in each pixel was calculated as

$$
\Delta F / F=\left[F_{\mathrm{c}}(x, y, t)-F_{\mathrm{av}}(x, y)\right] / F_{\mathrm{av}}(x, y)
$$

where $F_{\mathrm{av}}(x, y)$ is the average of $F_{\mathrm{c}}$ values calculated for data in 4-10 oscillatory cycles detected before the onset of stimulation. The average voltage change was considered to be linearly proportional to the change in the $-\Delta F / F$ value. In the present study, we obtained a stable $\Delta F / F$ value throughout each relatively long recording for $>40$ sec. To examine the influence of the estimation method on the results, we also calculated $\Delta F / F$ values based on a linear or a single exponential function. We found no distinctive differences among the images calculated using the three types of functions (data not shown).

We analyzed the activity of the PC lobe using the following four methods.

1. Real-time imaging of overall PC activity: The spatial distribution of $\Delta F / F$ values (PC activity) in each frame was reconstructed on a computer (Sun SS station 20) as an 8 -bit $128 \times 128$ pixel image according to a color table.

2. Serial plotting of local activity: For analysis of temporal changes in levels of local activity, $\Delta F / F$ values obtained from a $25(5 \times 5)$-pixel sq area were averaged in each frame and the spatially averaged activity levels were plotted serially in time.

3. Imaging of temporally averaged activity: For examination of the influence of olfactory stimulation on overall PC lobe activity, temporally averaged $\Delta F / F$ images were produced. First, a spatially averaged $\Delta F / F$ profile was obtained for a 25-pixel $(5 \times 5)$ area of the PC lobe, and then the timing of each depolarization peak was determined from the $\Delta F / F$ profile. Temporal averaging in each pixel was performed according to the timing of the peaks. In the present study, we obtained an average image by the averaging of $\Delta F / F$ values in three to five successive cycles in each pixel to increase the spatial signal-tonoise ratio.

4. Cross-correlation analysis: For identification of regions in which oscillatory activity was coherent within the PC lobe, cross-correlation analysis was performed. We calculated a spatially averaged activity profile for a $5 \times 5$ pixel area of the PC lobe as a reference wave and then calculated a correlation function between the reference wave and the signal obtained from each pixel. The area for the reference wave was selected from the apical part of the PC lobe, where the PC lobe was easily distinguished from meso- and metacerebral lobe by the shape. Based on each correlation function, the absolute maximum of the correlation coefficient $\left[C_{\max }(x, y)\right]$ for each pixel was determined. The distribution of $C_{\max }$ was very useful to clarify the border between the PC lobe and the other lobes when the PC lobe was indistinct in the optical images. In the present study, the PC lobe was identified based on the $C_{\max }$ distribution $\left(C_{\max }>0.3\right)$. Moreover, we calculated the time lag between the oscillation determined from each pixel and the reference oscillation using the cross-correlation functions.

\section{Results}

\section{SPONTANEOUS OSCILLATION OF PC LOBE ACTIVITY}

Morphologically, the PC lobe of Limax is divided into three regions: the cell, the terminal, and the internal masses (Zs.-Nagy and Sakharov 1970). The cell mass, which contains numerous cell bodies of PC interneurons, covers the posterior surface of the PC lobe (see Kimura et al. 1998b). Figure 1 shows typical results of optical measurement of the spontaneous activity of the hemiganglion from the posterior surface. The optical images clearly show the oscillatory activity of the PC lobe (Fig. 1a) with propagation of waves from the lateral to the medial borders (Fig. 1b). Simultaneous recording of the optical signals and the LFP revealed that the oscillatory changes in fluorescence intensity were synchronized to the oscillation in the LFP (Fig. 2, bottom). Based on optical signals recorded from the posterior surface of the PC lobe (Fig. 3a), we calculated the degree of correlation $\left[C_{\max }(x, y)\right]$ between the signal recorded from each pixel $(6 \times 6 \mu \mathrm{m})$ and a reference oscillation recorded in a small lateral area $(5 \times 5$ pixels $)$ of the $\mathrm{PC}$ lobe (Fig. 3b, open square). The $C_{\max }$ image (Fig. 3b) demonstrates that the activities recorded from every part of the PC lobe were strongly correlated to the reference wave. This indicates that oscillatory activity occurred throughout the PC lobe and that the oscillation in a local region was synchronized to that in other regions. The time lag between the signal in each pixel and the reference wave is also represented as a color image (Fig. 3c). This image, 

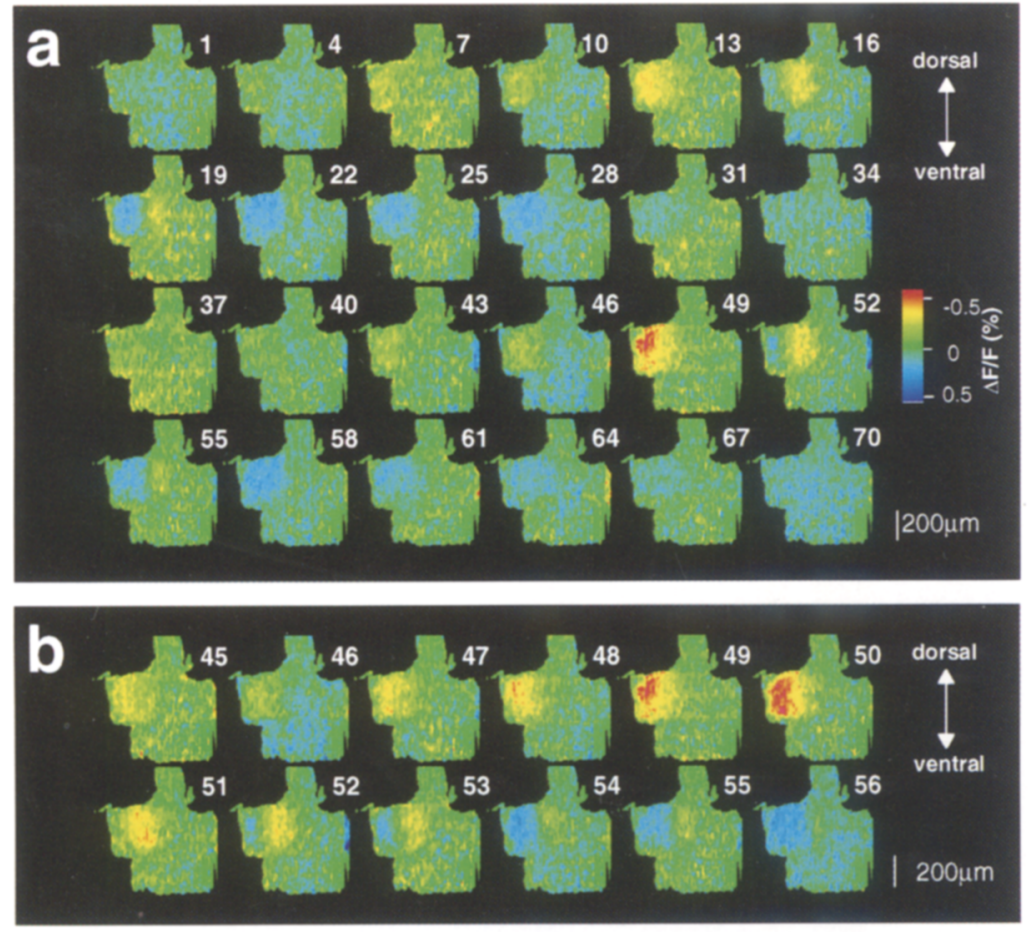

Figure 1: Spontaneous oscillation in the PC lobe. Each image was taken by accumulation of optical signals for $38.4 \mathrm{msec}$, and the $\Delta F / F$ value in each pixel is represented as a color image according to a color table; each frame is numbered. (a) Successive images showing the hemiganglial activity every $111.5 \mathrm{msec}$. Oscillatory activity was observed in the PC lobe. (b) High time resolution images showing propagation of an activity wave. obtained for the posterior surface, demonstrates that there was a time lag among the waves obtained from pixels along the lateral-medial (L-M) axis but not along the $\mathrm{D}-\mathrm{V}$ axis (Fig. 3c). These features of PC lobe oscillation are very similar to the PC lobe oscillation in L. maximus (Delaney et al. 1994). The wave velocity estimated from the result is $2.3 \mathrm{~mm} / \mathrm{sec}$.
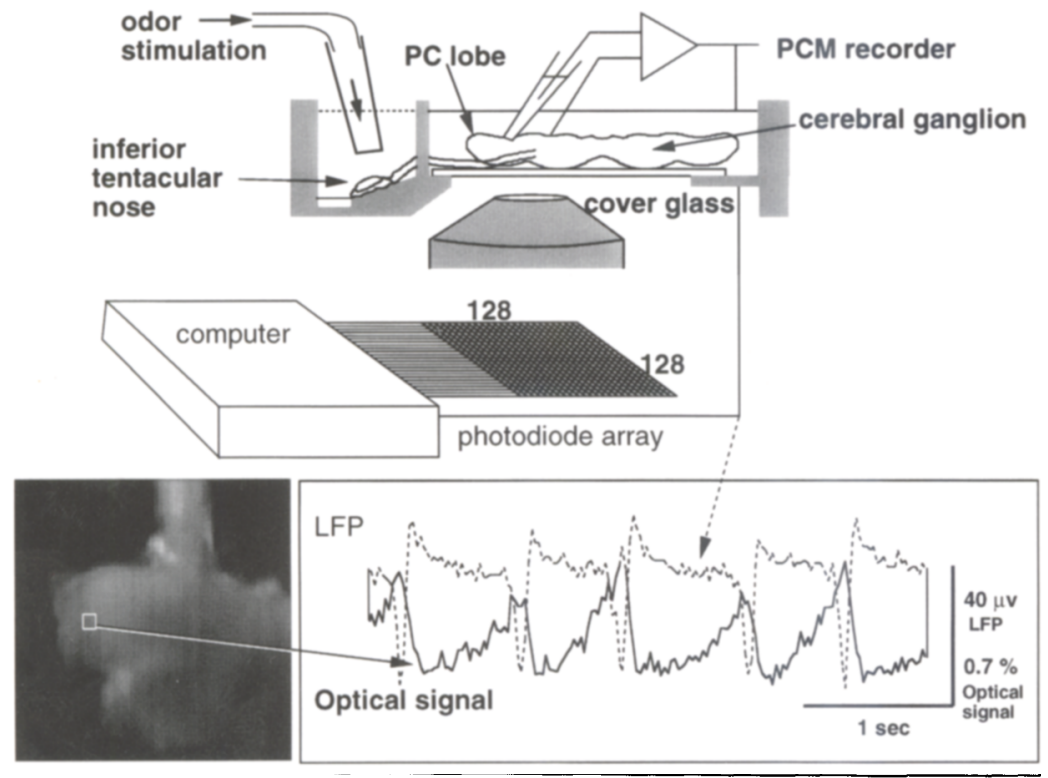

Figure 2: A schematic drawing showing the experimental setup (top) and recorded LFP and optical signals (bottom). The experimental chamber consisted of a nose and a ganglion compartment. The LFP of the PC lobe was recorded through a glass pipette of which the tip was placed on the anterior surface of the internal mass. Optical signals were recorded through a cover glass on the bottom of the ganglion bath. Odor application was performed after the olfactory epithelium was exposed to the air by changing the surface level of saline in the nose bath. The LFP and optical signals (bottom right) recorded from the PC lobe always showed oscillatory profiles, and the oscillations in LFP and $\Delta F / F$ were synchronized with each other. (Bottom left) A fluorescent view that was reconstructed from optical signals detected by the photodiode array. The time profile of optical signals (bottom right) was calculated from signals detected in the pixels shown as an open square in the image.

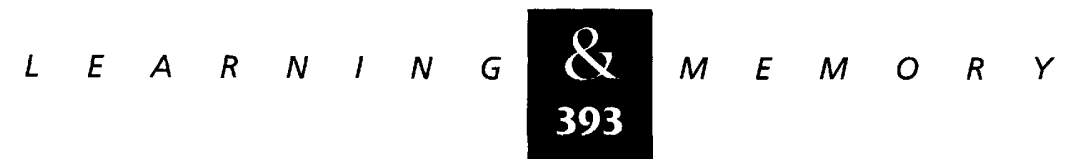


Kimura et al.

Figure 3: Results of correlation analysis of spontaneous oscillation. The activities of the PC lobes were recorded from the posterior $(a-c)$ and the dorsal $(d-f)$ surface. $(a, d)$ Fluorescence images; $(b, g)$ images showing correlation intensity in each pixel. The intensity was calculated from an optical signal obtained from each pixel and the reference oscillation obtained from a small area of the PC lobe (open squares in $b$ and $e$ ). $(c, f)$ Images showing the time lag of the oscillation recorded in each pixel with respect to the reference image.

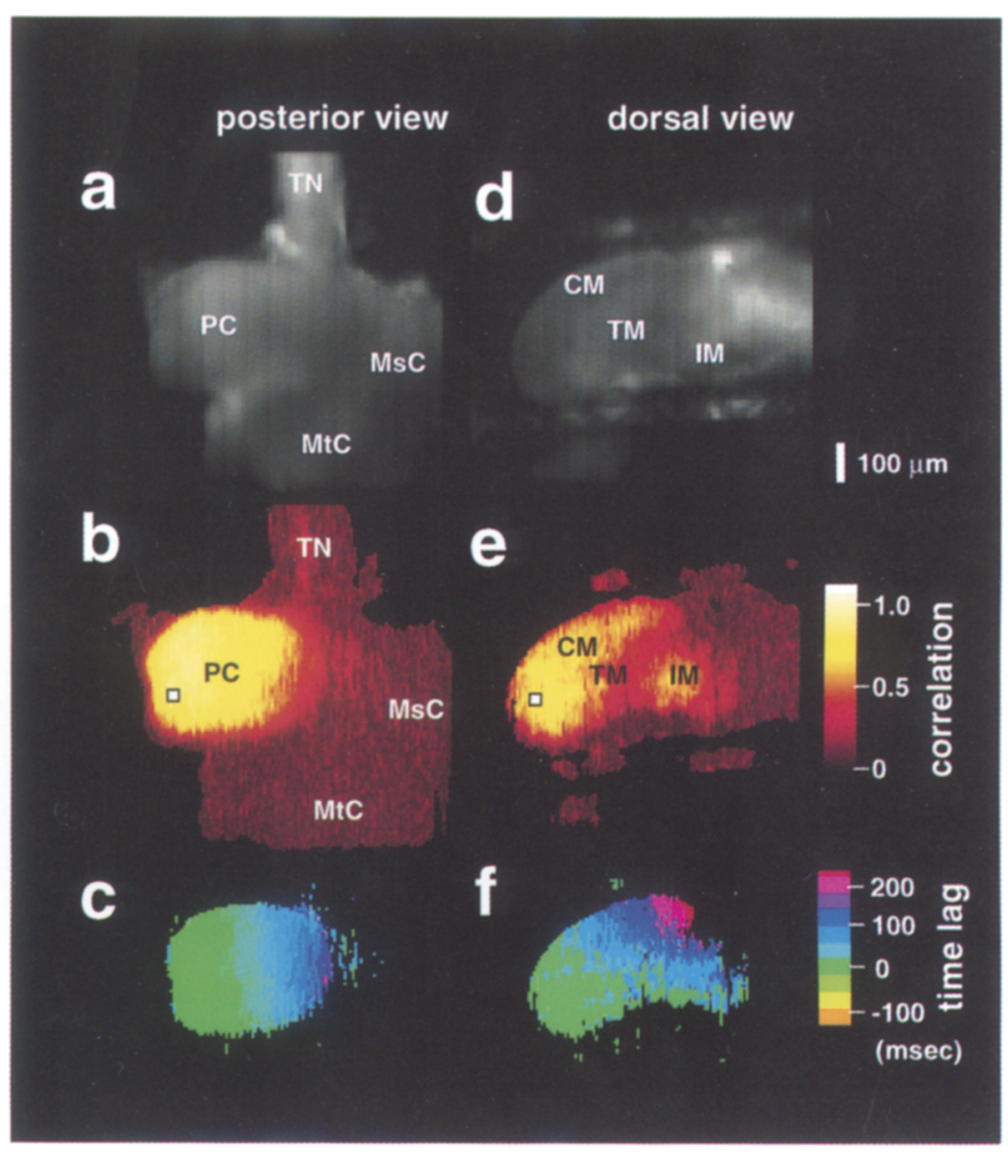

The same analysis was performed on optical recordings from the dorsal surface of the PC lobe. In these recordings, we could monitor the activities of the cell, terminal, and internal masses simultaneously (Fig. 3d). The correlation image showed that the oscillation occurred not only in the cell mass but also in the terminal and internal masses (Fig. 3e). The time lag image reveals that the time lag gradation also occurred not only in the cell mass but also in the terminal and internal masses (Fig. 3f). The origin of the PC lobe oscillation is in the PC interneurons (Delaney et al. 1994), and their neurites were extended to the terminal and internal masses (Kimura et al. 1998b). Thus, the spatial features of the arrangement of cell bodies in the cell mass may be maintained in their neurites in the terminal and internal masses. In addition, the activities in the terminal mass showed weaker correlation to the reference oscillation than those in the internal and cell masses (Fig. 3e). The terminal mass contains the terminals of projection fibers arising from the noses at the tentacle tips (Kimura et al. 1998b). Thus, the weakness of the correlation in the terminal mass may be caused by spontaneous activity of the projection neurons.

\section{ODOR-INDUCED MODULATION OF THE PC LOBE OSCILLATION}

We examined the response in brain-nose preparations that were isolated from two groups of slugs that had been subjected to differential conditioning and two-odor aversive conditioning. In the case of the differential-conditioned slugs, one of the odors had been used as a CS in the aversive conditioning (conditioned odor), and the other had been used as a CS in the unpaired training (unpaired odor). In the case of the two-odor-conditioned slugs, both odors were used as CSs in the aversive conditioning.

Figure 4 shows typical responses to carrot and cucumber odors in a preparation isolated from a differential-conditioned slug, which had been subjected to the aversive conditioning of the carrot odor and to the unpaired control treatment of the cucumber odor. As shown in the frequency pro- 


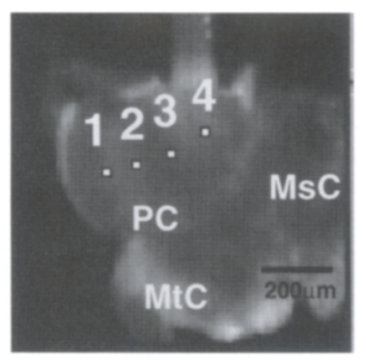

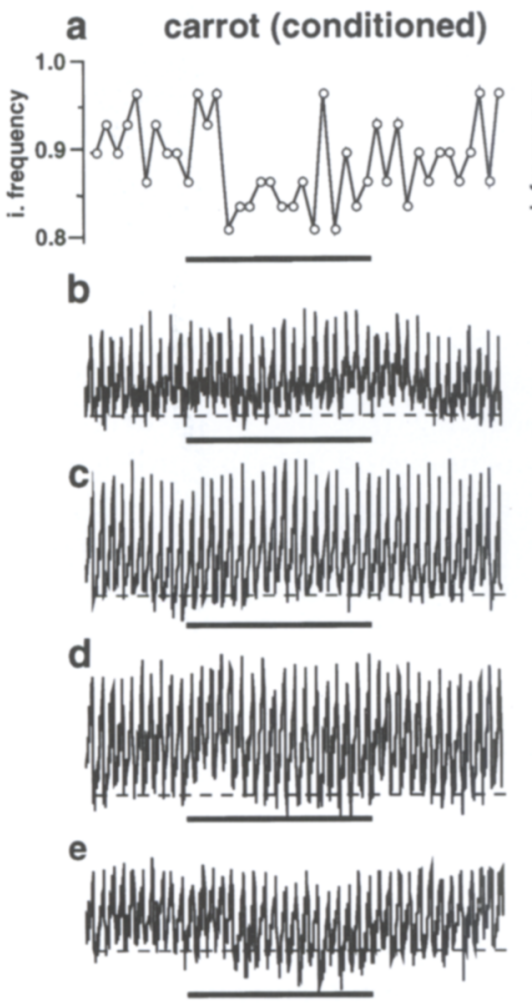

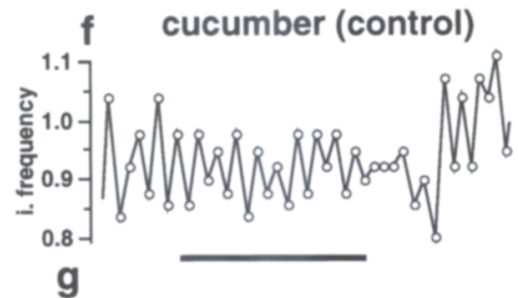

g
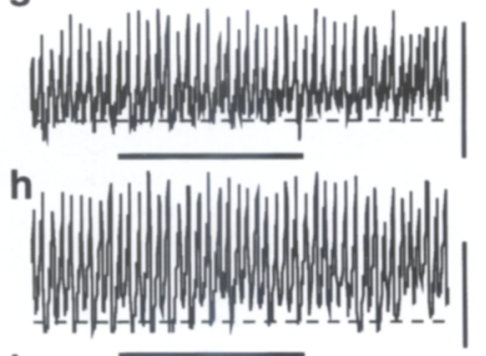

i

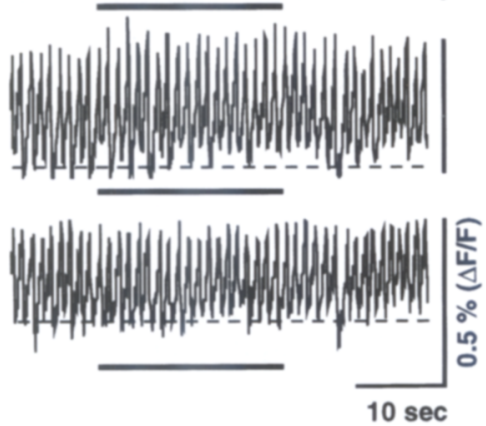

Figure 4: Olfactory responses in oscillations recorded in four different small regions of a PC lobe. The optical signals were recorded from the posterior surface of the hemiganglion. The regions in which optical signals were recorded are shown with the fluorescence image (top) as open squares ( $1-$ 4). The preparation was isolated from a differential-conditioned slug which had been subjected to aversive training with carrot odor and to unpaired training with cucumber odor. (a) Change in oscillation frequency induced by carrot odor; $(b-e)$ spatially averaged time profiles of $\Delta F / F$ in a response to carrot odor; $(f)$ change in oscillation frequency induced by cucumber odor; $(g-j)$ spatially averaged time profiles of $\Delta F / F$ in response to cucumber odor. The spatially averaged time profiles of $\Delta F / F$ in $b$ and $g$, in $c$ and $h$, in $d$ and $i$, and in $e$ and $j$ were calculated from signals recorded in regions $1,2,3$, and 4 , respectively (top). files, the instantaneous frequency was decreased -4 sec after the onset of carrot odor presentation (graph a), whereas no significant change in the instantaneous frequency occurred during cucumber odor presentation (graph $\mathrm{f}$ ). In both cases, optical signals were recorded from the PC lobe at a frame rate of $38.4 \mathrm{msec} /$ frame. The spatially averaged time profiles of $\Delta F / F$ calculated for four different areas of the posterior surface of the PC lobe are shown in Figure 4 (top, areas 1-4). Neither the carrot nor the cucumber odor induced a significant change in the optical signals (graphs b, c, g, and h) recorded in areas 1 and 2 of the PC lobe except for an increase in the cycle length of oscillation in response to carrot (graph a). The $\Delta F / F$ signals recorded in area 3 of the PC lobe show depolariza- tion in the basal potential (bottom level in each cycle of PC lobe oscillation) immediately after the onset of carrot odor presentation (graph d). On the other hand, the cucumber odor did not induce significant change in the oscillation recorded in the same area (graph i). In signals recorded from area 4 of the PC lobe, hyperpolarization in the basal potential occurred $-5 \mathrm{sec}$ after the onset of carrot odor stimulation (graph e) but not cucumber (graph j). These results demonstrate that the carrot odor induced at least two types of responses in different regions: fast and phasic depolarizing shift of the basal potential of the PC lobe oscillation, and slow and tonic hyperpolarizing shift of basal potential.

The odor-induced modulations of the basal po-

$$
\begin{array}{llllllllllllllll}
\hline & E & A & R & N & I & N & G & \bigotimes_{3} & M & E & M & O & R & Y
\end{array}
$$


tential in the PC lobe oscillations were accompanied by changes in amplitudes of the oscillation (see Fig 4, graphs d,e). The oscillation in the PC lobe was considered to reflect the membrane potential oscillation of PC interneurons (Delaney et al. 1994). Thus, it was considered that in the region where the modulation occurred PC interneurons changed their oscillatory properties upon presentation of the odor. For further examination, we produced temporally averaged $\Delta F / F$ images, which show the average level of neural membrane potential detected by each pixel for a period. For minimization of the influence of the oscillation on the averaging results, the temporal averaging was performed phase-locked to the oscillatory cycle (see Materials and Methods).

Figure 5 shows temporally averaged $\Delta F / F$ images calculated in 10 different segments of the two recordings shown in Figure 4 (periods $a-j$ in the upper graphs of Fig. 5). These temporally averaged $\Delta F / F$ images show that depolarization occurred especially in the neurons located in a belt-shaped region of the middle of the PC lobe immediately after the onset of the conditioned carrot odor presentation (red-colored region in image b). Then the depolarization was diminished (image c), and hyperpolarization occurred in the neurons in a relatively wide area of the PC lobe (blue-colored region in images $c$ and $d$ ). The region from which the time profile shown in Figure $4 \mathrm{~d}$ was obtained was contained in the belt-shaped region in Figure 5, image $\mathrm{d}$. Thus, it was confirmed that these temporally averaged $\Delta F / F$ images reflect the spatiotemporal changes in basal potential levels of PC lobe oscillations. On the other hand, when the cucumber odor was applied, the same preparation showed no significant change in the membrane potential in the early phase of the stimulation period (image g), although depolarization was observed in neurons located in a patch-shaped region in the late phase of the stimulation period (image i).

Figure 6 shows the responses to cucumber and carrot odors in a preparation isolated from a differential-conditioned slug that had been aversively conditioned to cucumber odor. In this preparation, the cucumber odor induced depolarization of basal potential levels of PC lobe oscillations in a beltshaped region (image a, arrow) in the early phase of the response, and then hyperpolarization of
Figure 5: Spatial and temporal properties of olfactory responses in the PC lobe. The two graphs (top) show responses in the oscillation frequency to the aversiveconditioned carrot (left graph) and unpaired control cucumber (right graph) odors; images (bottom) show temporally averaged $\Delta F / F$ images calculated from the optical recordings shown in Fig. 4. In the averaging, successive images in oscillatory cycle 3 or $4($; top) were averaged for four different periods $(a-h$, top). Images $a-h$ show the results of averaging in periods $a-h$. The aversiveconditioned carrot odor induced depolarization of basal potential levels of PC lobe oscillations in a belt-shaped region (b). The extent of the depolarization diminished rapidly (c), and the hyperpolarization of basal potential levels of PC lobe oscillations occurred $(c, d)$ according to frequency modulation (top left). When the unpaired control cucumber odor was applied to the inferior tentacular nose, no distinct modulation besides depolarization in a patch-shaped region in the latter phase of the response (i) was detected.

\section{carrot (conditioned)}
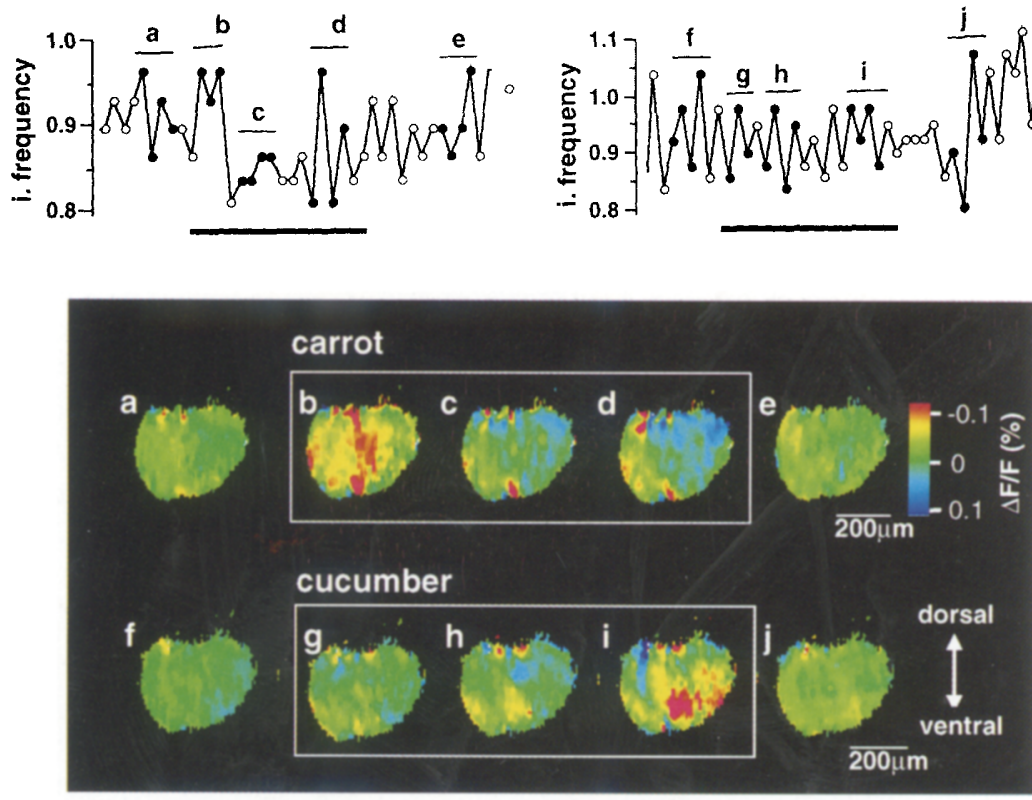

cucumber (control)

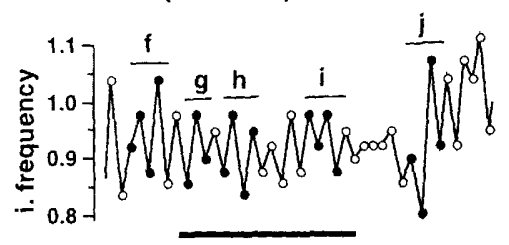

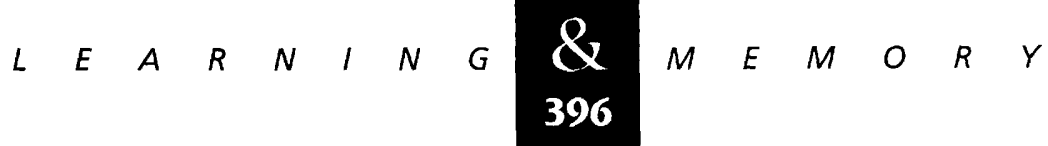



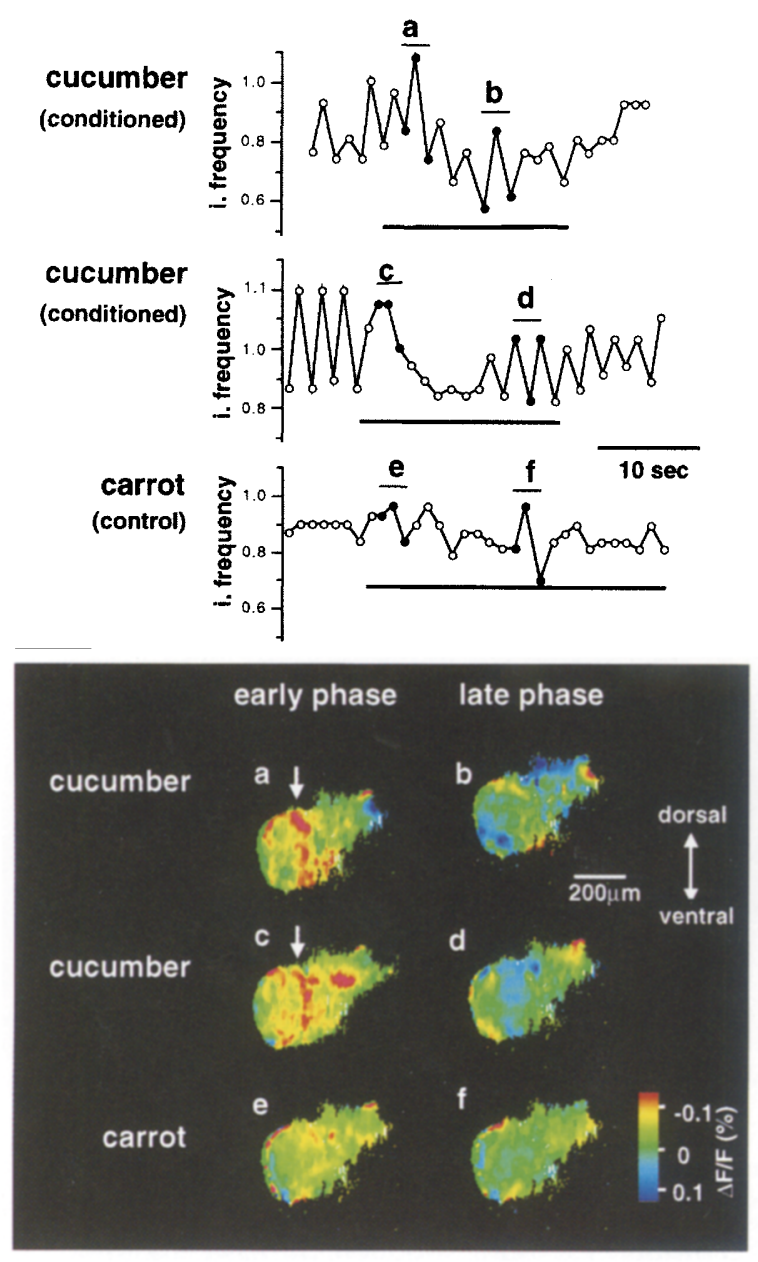

Figure 6: Spatial and temporal responses to odors in a preparation isolated from a differential-conditioned slug. The three graphs (top panel) show the responses in oscillation frequency to the aversive-conditioned cucumber (top and middle graphs) and the unpaired carrot (bottom graph) odors. (Bottom panel) The images (a-f) are averaged for periods $a-f$ in the graphs, respectively. After the onset of stimulation and before the frequency decrease occurred, depolarization of basal potential levels of PC lobe oscillations occurred, for both the first and the second application of the aversive-conditioned carrot odor, and after the start of frequency modulation hyperpolarization of basal potential levels of PC lobe oscillations occurred. Such changes in basal potential levels of PC lobe oscillations were not observed when the unpaired control cucumber odor was presented. Those records were obtained from the posterior surface of a preparation.

basal potential levels of PC lobe oscillations accompanied by frequency modulation (see top graph) occurred. The depolarization of basal potential levels of PC lobe oscillations in the belt-shaped region identified in the first trial was also detected in the second trial with cucumber odor (image c, arrow) but not in the patch-shaped region in image $c$. The pattern of spatiotemporal change of average membrane potential in PC lobes, which is characterized by depolarization in a belt-shaped region in the early phase of the response and by hyperpolarization of basal potential levels of PC lobe oscillations in a wide region of the PC lobe in the late phase of the odor response, was also similar to that induced by the conditioned carrot odor shown in Figure 5 (images a-e). On the other hand, neither the depolarization nor the hyperpolarization of basal potential levels of PC lobe oscillations was induced by the unpaired carrot odor (images $e$ and $f$ ).

In the present study, we examined the olfactory responses on 58 conditioned preparations and obtained 11 preparations in which a decrease of oscillation frequency was induced by a CS. In 9 of the 11 preparations, similar spatiotemporal modulations of basal potential level (early deporalization at narrow belt-shaped regions and late hyperporalization on a wide region of the PC lobe) were observed, whereas no distinctive response in basal potential levels was observed in the remaining preparations, which did not show response in oscillation frequency to conditioned odors. Therefore, it is considered that the spatiotemporal modulation pattern of the basal potential level of PC oscillation is involved in induction of frequency decrease of PC oscillation, which is specifically induced by aversively conditioned odors (Kimura et al. 1997a).

The responses of a preparation isolated from a two-odor-conditioned slug, which had been aversively conditioned to both carrot and cucumber odors, are shown in Figure 7 . The recordings were made from the dorsal surface of the PC lobe. The carrot odor induced the frequency decrease (top left graph) and depolarization of basal potential level of the PC lobe oscillations in a lateral region in the cell mass (image a, arrow). The cucumber odor also slightly decreased the frequency and induced depolarization of basal potential levels of PC lobe oscillations on the medial region of the cell mass (image $c$, arrow). Those responses in the cell mass were reproducibly observed in the second trial for each odor (data not shown). These results suggested that the region where depolarization in the cell mass occurred was specific to each conditioned odor. In the late phase, both odors induced hyperpolarization of basal potential levels of the PC lobe oscillations mainly in the terminal mass (images $\mathrm{d}$ and $\mathrm{b}$ ). 
Kimura et al.

Figure 7: Spatial and temporal responses to odors in a preparation isolated from a two-odor aversive-conditioned slug. The optical signals were recorded from the dorsal surface of the PC lobe. The two graphs (top) show the responses in the oscillatory frequency of the PC lobe to aversive-conditioned carrot (left) and cucumber (right) odors, and images a-d (bottom) were averaged for periods a-d shown in the graphs, respectively. Note that the odors induced depolarization of basal potential levels of PC lobe oscillations in different regions (arrows) in the early phase of the response (images $a$ and $c$ ). (CM) cell mass; (IM) internal mass; (MsC) mesocerebral lobe; (PC) procerebral lobe; (TM) terminal mass; (TN) tentacle nerve.
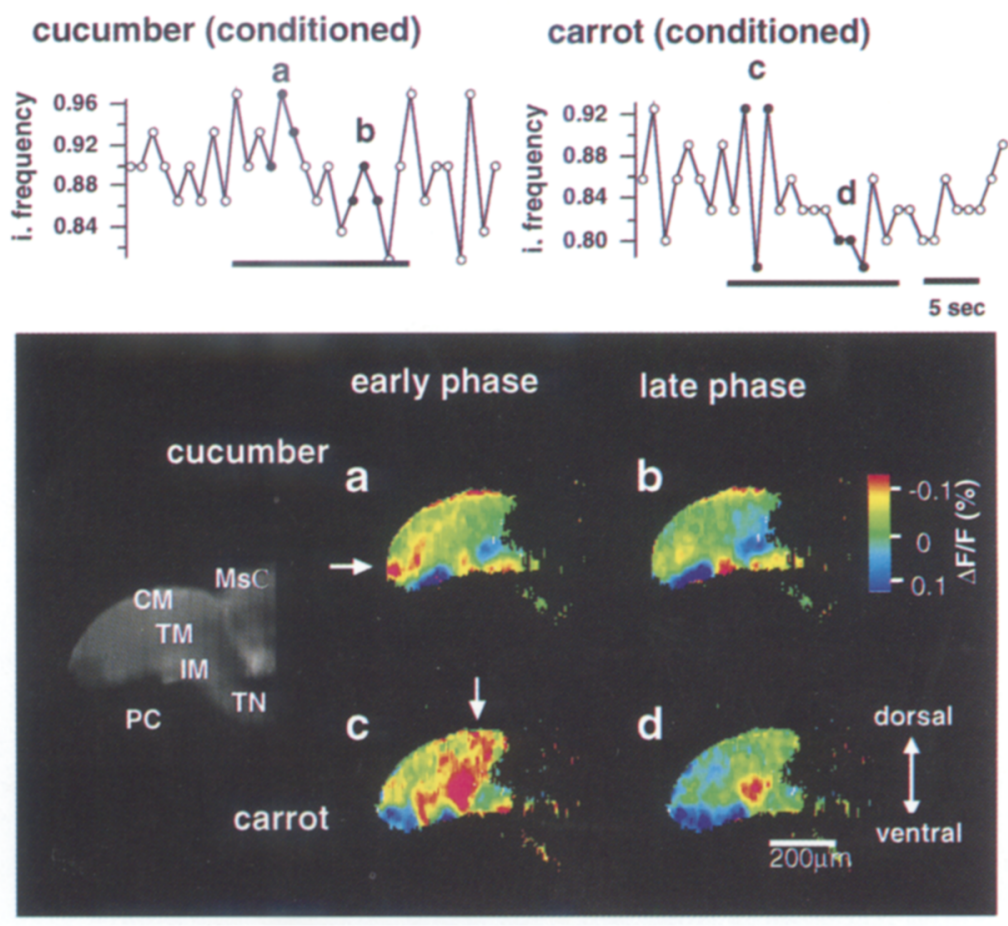

\section{Discussion}

In the present study, we found that aversively conditioned odors applied to the inferior tentacle tip evoked both depolarization and hyperpolarization in the basal membrane potential in PC interneurons. The depolarization of basal potential levels of PC lobe oscillations occurred immediately after the onset of odor stimulation and diminished after four or more cycles. Temporally averaged $\Delta F / F$ images showed that the depolarization occurred mainly in one or more belt-shaped regions in the PC lobe in the early phase of the response. After a decrease in depolarization, hyperpolarization of the basal potential levels of PC lobe oscillations was detected in a wider area of the PC lobe (see Fig. 5) and was sustained during stimulation. These results indicate that learned odors applied to the inferior tentacular nose produced a spatiotemporally patterned modulation of the basal potential levels of PC lobe oscillations.

The responses to unpaired odors were more obvious than those to conditioned odors. Unpaired odors usually did not induce modulation of basal potential level (Fig. 6), although we detected depolarization in a patch-shaped region in some cases (Fig. 5). The relation between such depolarization in a patch-shaped region and the odor response is unclear, because similar depolarization in a patch- shaped region was found to occur during spontaneous activity (data not shown).

The unpaired odors presented to the inferior nose basically did not modulate the basal potential levels in PC lobe oscillations. We also showed that a conditioned odor induced biphasic modulation of the basal potential levels of PC lobe oscillations, although the same preparations did not respond clearly to the unpaired control odors. The difference of the responses to the conditioned and the unpaired stimuli was not based on the type of conditioned odor (Figs. 5 and 6). Thus, it was considered that the biphasic modulation of the basal potential levels of neurons in PC lobe was specific to conditioned odors.

It was reported previously in L. maximus amyl that acetate, which is a natively repellent odor for the slug, applied to the superior tentacular nose induces a biphasic modulation of the PC lobe oscillation frequency (Gervais et al. 1996). Although the physiological meaning of the responses to such inputs to the superior tentacular nose is not yet clear (Gervais et al. 1996); this and our findings suggest that odor-induced responses in the PC lobe can be divided into at least two phases.

Kimura et al. (1998b) found that by using an activity marker, Lucifer yellow, the activity of $\leqslant 100$ PC interneurons in $L$. marginatus was enhanced after aversive conditioning, and that the 
labeled PC interneurons usually form a belt-shaped cluster along the D-V axis of the PC lobe (Kimura et al. 1998b). Two-trial conditioning performed using one odor resulted in detection of one belt-shaped cluster, and two-trial conditioning performed using two odors resulted in detection of two belt-shaped clusters. From these observations, it was concluded that enhancement of activity of a beltshaped cluster is related to formation of an odor memory. The present optical recording study results show that conditioned odors tended to induce depolarization of basal potential levels of PC lobe oscillations in a belt-shaped region of the PC lobe. Repeated stimulation with the same odor induced repeatedly depolarization of the basal potential levels of PC lobe oscillations in the same region (Fig. 6), and stimulation with a different odor induced depolarization in another region (Fig. 7). These features of the depolarization of basal potential levels of PC lobe oscillations detected in the early phase of olfactory responses suggest that the spatial patterns of the depolarization were related to the nature of the odor stimulus. The results of the present study and the experiments performed using the activity marker suggest that the PC lobe contains neurons whose activity is altered with production and recall of odor memories.

In behavior, slugs avoided the conditioned odor source with acute head turning, when it was presented $1 \mathrm{~cm}$ in front of the slugs' heads (Kimura et al. 1998a). We measured the interval between odor source presentation and acute head turning and found that the slugs started the turning with a delay of $4.8 \pm 3.7 \mathrm{sec}$ (mean \pm S.D., $n=20$ ) when carrot juice was presented, which had been used as CS (T. Kimura, unpubl.). On the other hand, in this study we demonstrate that the depolarization was detected only in $5 \mathrm{sec}$ or more (four or more cycles in PC lobe oscillation) after the onset of odor presentation, and then the hyperpolarization was detected. We speculate that events occurring in the PC lobe in the early phase of the olfactory response are necessary for odor recognition and behavioral determination.

Following depolarization, hyperpolarization of basal potential levels of PC lobe oscillations was observed in a wide area of the PC lobe during presentation of aversive-conditioned odors. The hyperpolarization was accompanied by a frequency modulation. Toda et al. (1996) found that the frequency of PC lobe oscillation is linearly correlated to the basal potential levels of neurons in Limax
PC lobe when modulation of the basal potential level occurs in a large area of the PC lobe. Thus, the induction of frequency modulation is related to induction of hyperpolarization in the late phase of the response. The nature of the modulation of oscillation frequency in the PC lobe is influenced by the learned preferences to the odor but not by the kind of odor (Kimura et al. 1998a). From these findings, we speculate that the activity of the PC lobe in the late phase of the response is involved in the result of recognition or behavioral determination that occurred in the early phase of the response to conditioned odors.

Spatiotemporal representation of information is widely considered to be a means of informational encoding in the brain, and some studies of olfactory networks in vertebrates and invertebrates have provided information on the manner of encoding. It was reported that an odor produces a spatially patterned neuronal activity gradient in the salamander olfactory bulb and the activity gradient changes temporally during stimulation (Cinelli et al. 1995).

The spatiotemporal change of activity in the olfactory bulb induced by an odor is considered to be constant among individuals (Cinelli et al. 1995), although it was reported that activities of olfactory bulb neurons are influenced by olfactory conditioning (Freeman and Schneider 1982). It was also reported that the olfactory neurons in the locust antennal lobe include a group of neurons that show oscillatory activities entrained with each other when an odor is sensed by the antennae (Laurent and Davidowitz 1994) and that the members of the group change temporally (Wehr and Laurent 1996). The spatiotemporal cell ensemble of the locust olfactory neurons is considered to be determined by the kinds of odors (Wehr and Laurent 1996).

In the present study, we also showed that presentation of conditioned odors applied to the inferior tentacular nose resulted in spatial and temporal changes in the activity of the $\mathrm{PC}$ lobe. The responses of the PC lobe to the inferior tentacular nose inputs were basically memory-related ones. Although the manner of representation of learned odors in the PC lobe cannot be directly compared to those in the olfactory network in other animals, the findings of the present study and in other olfactory systems suggest that spatiotemporal representation of information was employed as a general manner of encoding in some levels of olfactory processing. 


\section{Acknowledgments}

We thank Alan Gelperin for comments on the manuscript. A part of this study was performed through Special Coordination Funds of the Science and Technology Agency and was supported by a grant from Promotion of Basic Research Activities for Innovative Biosciences and by grants-in-aid for Scientific Research (0729105 and 07557143 to Y.K.) from the Minister of Education, Science, Sports, and Culture, Japan.

The publication costs of this article were defrayed in part by payment of page charges. This article must therefore be hereby marked "advertisement" in accordance with 18 USC section 1734 solely to indicate this fact.

\section{References}

Cinelli, A.R., K.A. Hamilton, and J.S. Kauer. 1995. Salamander olfactory bulb neural activity observed by video-rate voltage-sensitive dye imaging. 3 . Spatial and temporal properties of responses evoked by odorant stimulation. J. Neurophysiol. 73: 2053-2071.

Delaney, K., A. Gelperin, M.S. Fee, J.A. Flores, R. Gervais, D.W. Tank, and D. Kleinfeld. 1994. Waves and stimulus-modulated dynamics in an oscillating olfactory network. Proc. Natl. Acad. Sci. 91: 669-673.

Freeman, W.J. and W.S. Schneider. 1982. Change in spatial patterns of rabbit olfactory EEG with conditioning. Psychophysiology 19: 44-56.

Gelperin, A. and D.W. Tank. 1990. Odor-modulated collective network oscillation of olfactory interneurons in a terrestrial mollusc. Nature 345: 437-440.

Gelperin, A., L. Rhines, J. Flores, and D.W. Tank. 1993. Coherent network oscillations of olfactory interneurons: Modulation by endogenous amines. J. Neurophysiol. 69: 1930-1939.

Gervais, A., K. Kleinfeld, R. Delaney, and A. Gelperin. 1996. Central and reflex responses elicited by odor in a terrestrial mollusk. J. Neurophysiol. 76: 1327-1336.

Gray, C.M., P. Konig, A.K. Engek, and W. Singer. 1989. Oscillatory responses in cat visual cortex exhibit inter-columnar synchronization which reflects global stimulus properties. Nature 338: 334-337.

Kawahara, S., S. Toda, Y. Suzuki, S. Watanabe, and Y. Krino. 1997. Comparative study in neural oscillation in the procerebrum of the terrestrial slugs Incilaria bilineata and Limax marginatus. J. Exp. Biol. 200: 1851-1861.

Kimura, T., S. Toda, T. Sekiguchi, and Y. Kirino. 1998a Behavioral modulation induced by food odor aversive conditioning, and its influence on the response of an oscillatory brain network in the slug, Limax marginatus. Learn. \& Mem. (this issue).

Kimura, T., H. Suzuki, E. Kono, and T. Sekiguchi. 1998b.
Mapping of interneurons which contribute to food aversive conditioning in the slug brain. Learn. \& Mem. (this issue).

Kleinteld, D., D.E. Delaney, M.S. Fee, J.A. Flores, D.A. Tank, and A. Gelperin. 1994. Dynamic of propagating waves in the olfactory network of a terrestrial mollusk: An electrical and optical study. J. Neurophysiol. 72: 1403-1419.

Laurent, G. and H. Davidowitz. 1994. Encoding of olfactory information with oscillating neural assemblies. Science 265: 1872-1875.

Laurent, G. and M. Naragi. 1994. Odorant-induced oscillations in the mushroom bodies of the locust. J. Neurosci. 14: 2993-3004.

Laurent, G., M. Wehr, and H. Davidowitz. 1996. Temporal representation of odors in an olfactory network. J. Neurosci. 16: $3837-3847$

Toda, S., T. Kimura, S. Kawahara, T. Sekiguchi, and Y. Kirino. 1996. Optical study of central olfactory processing on the terrestrial slug. Neurosci. Res. (Suppl.) 20: S223.

Wehr, M. and G. Laurent. 1996. Odour encoding by temporal sequences of firing in oscillating neural assembles. Nature 384: 162-165.

Yamada, A., H. Suzuki, T. Sekiguchi, and A. Mizukami. 1992. Behavioral analysis of internal memory state using cooling induced retrograde amnesia in Limax flavus. J. Neurosci. 12: 729-735

Zs.-Nagy, I. and D.A. Sakharov. 1970. The fine structure of the procerebrum of pulmonate molluscs, Helix and Limax. Tissue Cell 2: 399-411.

Received May 23, 1997; accepted in revised form January 12, 1998. 


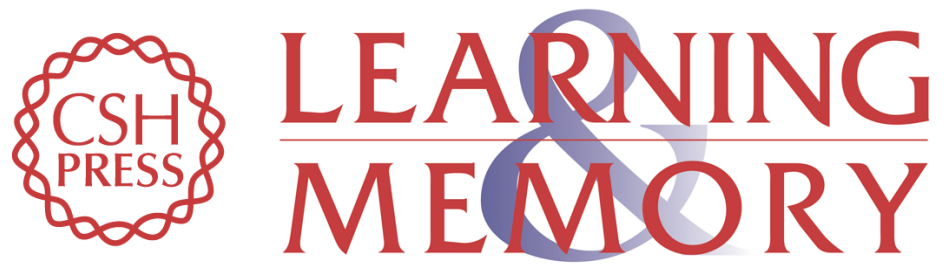

\section{Optical recording analysis of olfactory response of the procerebral lobe in the slug brain.}

T Kimura, S Toda, T Sekiguchi, et al.

Learn. Mem. 1998, 4:

Access the most recent version at doi:10.1101//m.4.5.389

References This article cites 16 articles, 6 of which can be accessed free at:

http://learnmem.cshlp.org/content/4/5/389.full.html\#ref-list-1

License

Email Alerting Receive free email alerts when new articles cite this article - sign up in the box at the Service top right corner of the article or click here. 